\section{Liquids Section of the CMD}

The European Physical Society recently decided to create a Liquids Section as part of its Condensed Matter Division. The decision was motivated by the vitality of this branch of science and by its multiple facets which encompass activities varying from basic statistical mechanics to chemical engineering. The suggestion for the creation of such a Section originated from a number of scientists working in this broad field who deplored the lack of coordination. Council considered the initiative to be timely and approved it at its Session in Winterthur in March 1984.

For two centuries, the history of liquid state physics coincided with that of fluid mechanics. Its development was intimately connected with the prestigious names, known to all physicists, of Euler, Navier, Stokes and Prandtl. Its extension to chemically reacting fluids, much more recently, is due to Bodenstein, Damköhler, Von Karman, Prigogine and many others. This direction of research was greatly stimulated by practical problems of chemical engineering and of space research. Liquid state physics took a new direction when, thirty years ago, modern methods of statistical mechanics were applied to study microscopic properties of liquids. The development of this field, in which powerful computer simulation methods took an important part, was exceptionally fast and personalities such as Yvon, Kirkwood, Kubo, Van Hove and Adler contributed significantly to it. Finally, liquid crystals, recognized by $R$. Friedel as a new organization of matter, have been investigated succesfully by Franck, Gray, de Gennes and many other scientists.

A particular consequence of this evolution is the non-existence of a coherent community of liquid state physicists. Its members, engineers, physicists and chemists, live in different departments, read different journals and attend different meetings. This situation, in fact, has its origin in the physics itself. Macroscopic and microscopic processes evolve over vastly different time and length scales and are thus de-cor-related to a considerable extent. Nevertheless, there are processes in which these scales are such that various mechanisms belonging, not only to fluid and statistical mechanics, but also to non-linear physics are intimately connected. Transitions between laminar and turbulent flows and processes like colloidal dispersions are two well known recent examples of this type. Their study is certainly slowed down by the absence of unity of the Liquid State community.

The structure of the Section is conceived to provide an answer. The essential task of the Section is to promote the study of liquids on a broad basis, to arrange scientific meetings and to help the existing European organizations. The following topics are included in the programme:
(1) Microscopic physics of liquids (statistical mechanics, computer simulation, optical and picosecond laser spectroscopies, neutron scattering).

(2) Fluid mechanics (instabilities, transition to turbulence, fully developed turbulence and chaos).

(3) Chemically reacting fluids (reaction-diffusion processes near and out of equilibrium, periodic and chaotic behaviour, laminar turbulent reacting flows).

(4) Organized liquids (thermotropic and lyotropic mesophases, flexible and semi-rigid polymer solutions).

(5) Semi-macroscopic random matter (colloïds, flows in porous media, aggregates, granular matter and composites, gels).

(6) Dispersed media and non-Newtonian liquids (rheology of dispersed media, viscoelastic flows).

It can be seen from the programme that much remains to be done. Let us hope that European scientists will participate in this effort and that the creation of the Liquids Section will accelerate it!

S. Bratos

(Chairman, Liquids Section Committee)

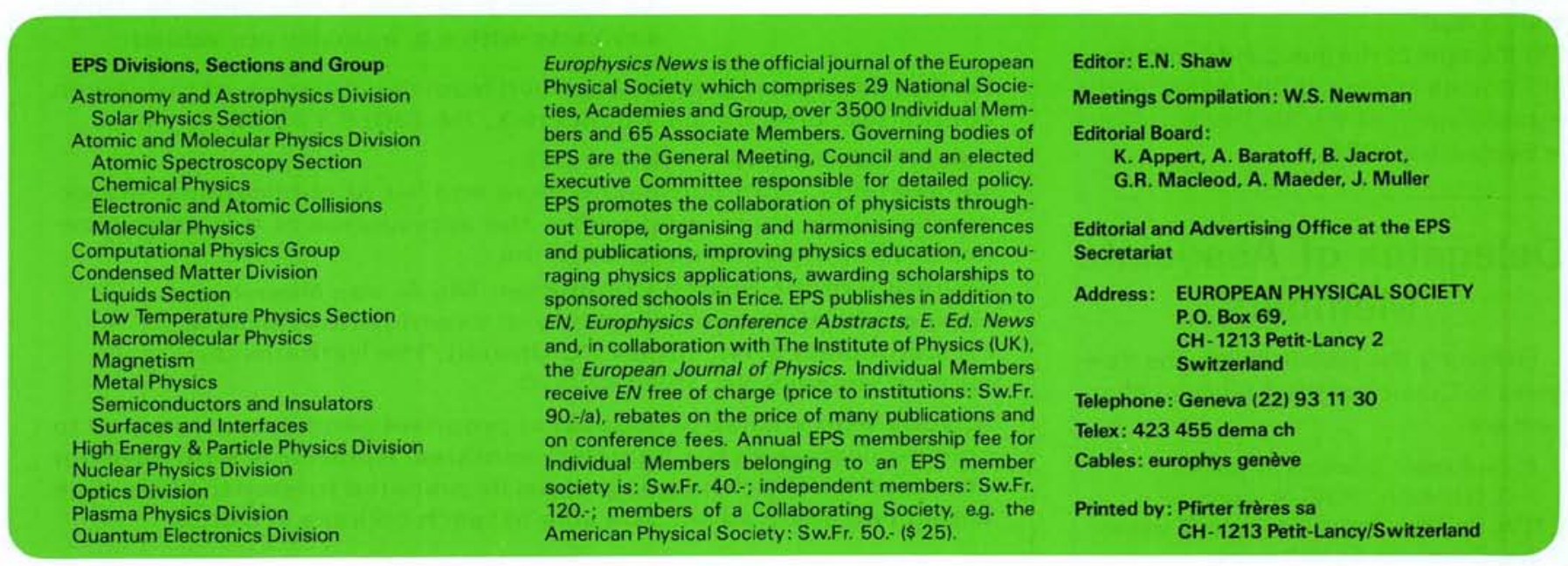

Applications are invited for the post of Lecturer in Physics in the general area of liquid state physics with an interest in the interplay between statistical mechanical theory and experiment. Experience in some aspect of experimental liquid state physics or computer simulation would be an advantage but is not essential. The successful candidate will, however, be expected to be associated with the work of Professor J.E. Enderby or Dr. R.E. Evans. Salary will be on the Lecturers' salary scale ( $f$ 7,190 — $f$ 14,125), under review.

Further particulars should be obtained from the : Registrar and Secretary,

University of Bristol,

Senate House, Bristol BS8 1TH,

to whom applications should be sent by 27 July 1984, or as soon as possible thereafter, quoting reference $\mathrm{JC}$. 